\title{
Lentivirus-induced knockdown of LRP1 induces osteoarthritic-like effects and increases susceptibility to apoptosis in chondrocytes via the nuclear factor- $\kappa B$ pathway
}

\author{
ERPING YANG $^{1 *}$, HUIFENG ZHENG $^{1 *}, \mathrm{HAO} P E N G^{1}$ and YINYUAN DING ${ }^{2}$ \\ ${ }^{1}$ Department of Orthopedics, Renmin Hospital of Wuhan University, Wuhan, Hubei 430060, P.R. China; \\ ${ }^{2}$ Department of Molecular Genetics, University of Texas Southwestern Medical Center, Dallas, TX 75390, USA
}

Received July 30, 2014; Accepted April 17, 2015

DOI: $10.3892 /$ etm.2015.2471

\begin{abstract}
Low-density lipoprotein receptor-related protein 1 (LRP1) is known to regulate cell survival and inflammation. The present study investigated the involvement of LRP1 in the regulation of tumor necrosis factor (TNF)- $\alpha$-induced expression of matrix metalloproteinase (MMP)-13. Furthermore, the study aimed to elucidate the mechanisms underlying the effects of LRP1 on TNF- $\alpha$-induced inflammation and apoptosis of chondrocytes. Lentivirus-mediated RNA interference techniques were used to knockdown the LRP1 gene. Subsequently, the effects of LRP1 on TNF- $\alpha$-induced MMP-13 expression were determined using quantitative polymerase chain reaction, western blot analysis and ELISA. Furthermore, the TNF- $\alpha$-induced intracellular pathway was investigated using a nuclear factor (NF) $\kappa \mathrm{B}$ inhibitor (Bay 11-7082). In addition, the effect of LRP1 regulation on growth and apoptosis in chondrocytes was investigated using western blot analysis and a TUNEL assay. LRP1 knockdown was shown to increase TNF- $\alpha$-induced MMP-13 expression via the activation of the NF- $\kappa \mathrm{B}$ (p65) pathway, which reduced the expression of collagen type II and cell viability. In addition, LRP1 inhibited cell apoptosis by increasing the expression of phospho-Akt and B-cell lymphoma 2 (Bcl-2), while suppressing the expression of caspase- 3 and Bcl-2-associated $\mathrm{X}$ protein. The results of the present study indicated that LRP1 was able to inhibit TNF- $\alpha$-induced apoptosis and inflammation in chondrocytes. Therefore, LRP1 may be an effective osteoarthritis inhibitor,
\end{abstract}

Correspondence to: Professor Hao Peng, Department of Orthopedics, Renmin Hospital of Wuhan University, 99 Zhangzhidong Road, Wuhan, Hubei 430060, P.R. China

E-mail: penghao5868@hotmail.com

${ }^{*}$ Contributed equally

Key words: tumor necrosis factor- $\alpha$, matrix metalloproteinase-13, low-density lipoprotein receptor-related protein-1, apoptosis, osteoarthritis potentially providing a novel approach for antiarthritic therapeutics.

\section{Introduction}

Osteoarthritis (OA) refers to a group of degenerative joint diseases, which may be initiated by joint injuries, obesity, gene mutations and aging. Cartilage extracellular matrix (ECM) is maintained by chondrocytes, which synthesize aggrecan and collagen, providing mechanical strength and flexibility to joints. Osteoarthritic chondrocytes exhibit an altered metabolism and an imbalance between anabolic growth factors and proinflammatory cytokines, including tumor necrosis factor (TNF)- $\alpha$ and interleukin (IL)-1, which are produced by inflammatory cells, synovial fibroblasts and chondrocytes. Chondrocytes are a distinct cell type that are targets for proinflammatory cytokines during the pathogenesis of OA. These cytokines induce the expression of matrix metalloproteinases (MMPs), which subsequently cleave various components of the ECM (1-4). However, the mechanisms by which TNF- $\alpha$ increases the expression of MMP-13 in chondrocytes are yet to be fully elucidated.

Previous studies have indicated that low-density lipoprotein receptor-related protein 1 (LRP1) may regulate cell survival (5) and inflammation (6). LRP1 is hypothesized to regulate lipid homeostasis, extracellular proteolysis, growth factor activity, the composition of the ECM and immune responses (7). A significant number of these LRP1 functions are considered to be associated with endocytosis and cellular signal transduction pathways $(8,9)$. Overton et al (10) proposed that the activity of LRP1 in atherosclerosis may be associated with its ability to suppress local inflammation. Furthermore, LRP1 suppresses the expression of inflammatory mediators indirectly via the regulation of TNF receptor 1 (TNFR1)-dependent cell signaling through the I $\kappa \mathrm{B}$ kinase nuclear factor (IKK-NF) $-\kappa \mathrm{B}$ pathway (11). Similarly, Gaultier et al (12) reported that LRP1 may inhibit inflammation in cases of peripheral nerve injury.

However, the mechanisms by which LRP1 regulates inflammation and apoptosis in chondrocytes remain unclear. In the present study, it was hypothesized that LRP1 protected chondrocytes against apoptosis and TNF- $\alpha$-induced inflammation. Therefore, the aim of the present study was to define 
the role of LRP1 in TNF- $\alpha$-induced apoptosis and inflammation of articular chondrocytes in vitro. Thus, the mechanisms may identify a novel target for attenuating catabolic TNF- $\alpha$ activity in cartilage tissue.

\section{Materials and methods}

Antibodies and reagents. Rabbit monoclonal antibodies targeting LRP1 (\#2703-1; 1:1,000) were obtained from Epitomics, Inc. (Burlingame, CA, USA), while monoclonal rabbit antibodies against glyceraldehyde 3-phosphate dehydrogenase (GAPDH; \#2118; 1:1,000), phosphorylated NF-кB subunit p65/RelA (\#3033; 1:1,000) and mouse monoclonal antibodies against IкB (\#4814; 1:1,000) and were purchased from Cell Signaling Technology, Inc. (Danvers, MA, USA). In addition, rabbit polyclonal antibodies targeting Akt (\#BS2987; 1:700), phospho (p)-Akt (\#BS4009; 1:600), B-cell lymphoma 2 (Bcl-2; \#BS1511; 1:600), Bcl-2-associated X protein (Bax; \#BS1030; 1:600) and caspase-3 (\#BS1518; 1:700) were purchased from Bioworld Technology, Inc. (St. Louis Park, MN, USA), and rabbit polyclonal antibodies against MMP-13 (\#sc-30073; 1:600) and nitric oxide synthase (iNOS; \#sc-67003; 1:600) were purchased from Santa Cruz Biotechnology, Inc. (Dallas, TX, USA). Dulbecco's modified Eagle's medium (DMEM)/F-12 nutrient mixture and fetal bovine serum (FBS) were purchased from Gibco Life Technologies (Carlsbad, CA, USA). Horseradish peroxidase (HRP)-conjugated anti-rabbit IgG and anti-goat IgG secondary antibodies were purchased from Wuhan Boster Biological Technology, Ltd. (Wuhan, China). Recombinant rat TNF- $\alpha$ was purchased from PeproTech, Inc. (Rocky Hill, NJ, USA). An NF- $\kappa$ B inhibitor (Bay 11-7082) was purchased from the Beyotime Institute of Biotechnology (Haimen, China). This study was approved by the Ethics Committee of Wuhan University (Wuhan, China).

Cell lines. Primary chondrocytes were isolated from the knee joint of rats (age, 7 days) using a sequential proteinase and collagenase digestion technique (13), and the cells released by enzymatic digestion were cultured to a density of 3.0x $10^{6}$ cells/ml in DMEM/Ham's F-12 media (1:1) supplemented with $10 \%$ FBS (complete media). The cells were cultured at $37^{\circ} \mathrm{C}$ in humidified air with $5 \% \mathrm{CO}_{2}$. The cells were maintained at $20-80 \%$ confluency, and cells between passages 2 and 3 were used for the following experiments. Subsequently, the cells were plated onto a six-well plate at a density of $2.0 \times 10^{5}$ cells $/ \mathrm{ml}$. The chondrocytes were starved in DMEM overnight, after which pretreatment with the Bay 11-7082 NF- $\kappa \mathrm{B}$ inhibitor $(10 \mu \mathrm{M})$ was conducted 30 min prior to the addition of TNF- $\alpha$. With regard to the signaling experiments, chondrocytes were treated with TNF- $\alpha$ (30 $\mathrm{ng} / \mathrm{ml}$ ) for $30 \mathrm{~min}$. For the quantitative polymerase chain reaction (qPCR) experiments, the cells were treated with TNF- $\alpha(30 \mathrm{ng} / \mathrm{ml})$ for $12 \mathrm{~h}$.

Construction of a lentiviral vector expressing LRP1-targeting small hairpin (sh)RNA. Knockdown of LRP1 expression in the chondrocytes was achieved using a lentivirus vector expressing a rat LRP1-targeting shRNA (shLRP1; Genechem, Shanghai, China), according to the manufacturer's instructions. The target nucleotide sequence of the oligoduplexes was as follows: 5'-GCAUUGGCGUGCAGCUUAAUU-3'. A non-targeting control (NTC) oligoduplex was used as a negative control. At a multiplicity of infection of 20:1 for $12 \mathrm{~h}$ after transfection, the medium was exchanged for fresh medium, and the cells were cultured for a further 48-72 h. mRNA was harvested after $48 \mathrm{~h}$ and protein was harvested after $72 \mathrm{~h}$. The effects of LRP1 knockdown on the expression of various indices were determined using qPCR and western blot analysis.

Western blot analysis. Protein was extracted from stably transfected cells using a radioimmunoprecipitation assay lysis buffer (Beyotime Institute of Biotechnology), according to the manufacturer's instructions. The protein concentration was measured using a bicinchoninic acid protein assay kit (Beyotime Institute of Biotechnology), after which $30 \mu \mathrm{g}$ protein per lane was loaded onto a $10 \%$ SDS-PAGE gel. Proteins were transferred to a polyvinylidene difluoride membrane, and blocked with 5\% non-fat dry milk in Tris-buffered saline and Tween 20 (TBST). The membranes were incubated with the primary antibodies overnight at $4^{\circ} \mathrm{C}$, and subsequently washed three times with TBST. Next, the membranes were incubated with the HRP-conjugated secondary antibodies $(1: 5,000)$ in TBST for $2 \mathrm{~h}$ at room temperature. After washing with TBST buffer, the western blots were visualized using an enhanced chemiluminescence system (Thermo Fisher Scientific, Waltham, MA, USA), and exposed to Kodak X-ray film (Kodak, Rochester, NY, USA).

Reverse transcription $(R T)-q P C R$. Total RNA was extracted using TRIzol reagent (Invitrogen Life Technologies, Carlsbad, CA, USA), according to the manufacturer's instructions. First-strand cDNA synthesis was conducted using an RT-PCR kit (Takara Biotechnology Co., Ltd., Dalian, China), according to the manufacturer's instructions. Target gene mRNA expression levels were determined using a SYBR Premix Ex Taq (Takara Biotechnology Co., Ltd.) qPCR system, with the following protocol: Denaturation for $10 \mathrm{sec}$ at $95^{\circ} \mathrm{C}$, followed by 40 cycles of $15 \mathrm{sec}$ at $95^{\circ} \mathrm{C}$ and $60 \mathrm{sec}$ at $60^{\circ} \mathrm{C}$. Forward and reverse primer sequences are presented in Table I. GAPDH from the same sample was used as the internal control, and the relative contents of the copy numbers of the target gene mRNA were calculated. The qPCR experiments were conducted independently and in triplicate.

Determination of the levels of $M M P-13$ and collagen type II $\alpha 1$ (COL2A1) using ELISA. Control and shLRP1 chondrocytes were pretreated with Bay 11-7082 $(10 \mu \mathrm{M})$ for $2 \mathrm{~h}$ and incubated with or without TNF- $\alpha(30 \mathrm{ng} / \mathrm{ml})$ for a further $24 \mathrm{~h}$. The effect of TNF- $\alpha$ on the expression levels of MMP-13 and COL2A1 secreted by the chondrocytes in the culture medium was assessed using a rat MMP-13 and COL2A1 ELISA kit (Wuhan Elabscience Biotechnology Co., Ltd., Wuhan, China), according to the manufacturer's instructions.

TUNEL assay. Control and shLRP1 chondrocytes were incubated with or without TNF- $\alpha(30 \mathrm{ng} / \mathrm{ml})$ for $24 \mathrm{~h}$. Apoptotic cells were detected using a TUNEL assay (Roche Diagnostics, Basel, Switzerland), according to the manufacturer's instruc- 
Table I. Oligonucleotide primers used for quantitative polymerase chain reaction analysis.

\begin{tabular}{lcc}
\hline Gene & Accession number & Primer sequence \\
\hline GAPDH & & \\
Forward & NC_005103.3 & ACAGCAACAGGGTGGTGGAC \\
Reverse & & TTTGAGGGTGCAGCGAACTT \\
LRP1 & NM_001130490 & GACTAACCCCTGTGACCGCAA \\
Forward & & GGTGCCATTGTCCAGCCTCTT \\
Reverse & & \\
TNFR1 & NC_005103.3 & TCAAAGAGGTGGAGGGTGAAGGA \\
Forward & & AGACAGGATGACTGAAGCGTGGG \\
Reverse & & \\
iNOS & NC_005109.3 & AAAATGGTTTCCCCCAGTTC \\
Forward & & GCTTGTCTCTGGGTCCTCTG \\
Reverse & & \\
\hline
\end{tabular}

GAPDH, glyceraldehyde 3-phosphate dehydrogenase; LRP1, low-density lipoprotein receptor-related protein 1; TNFR1, tumor necrosis factor receptor 1 ; iNOS, nitric oxide synthase.

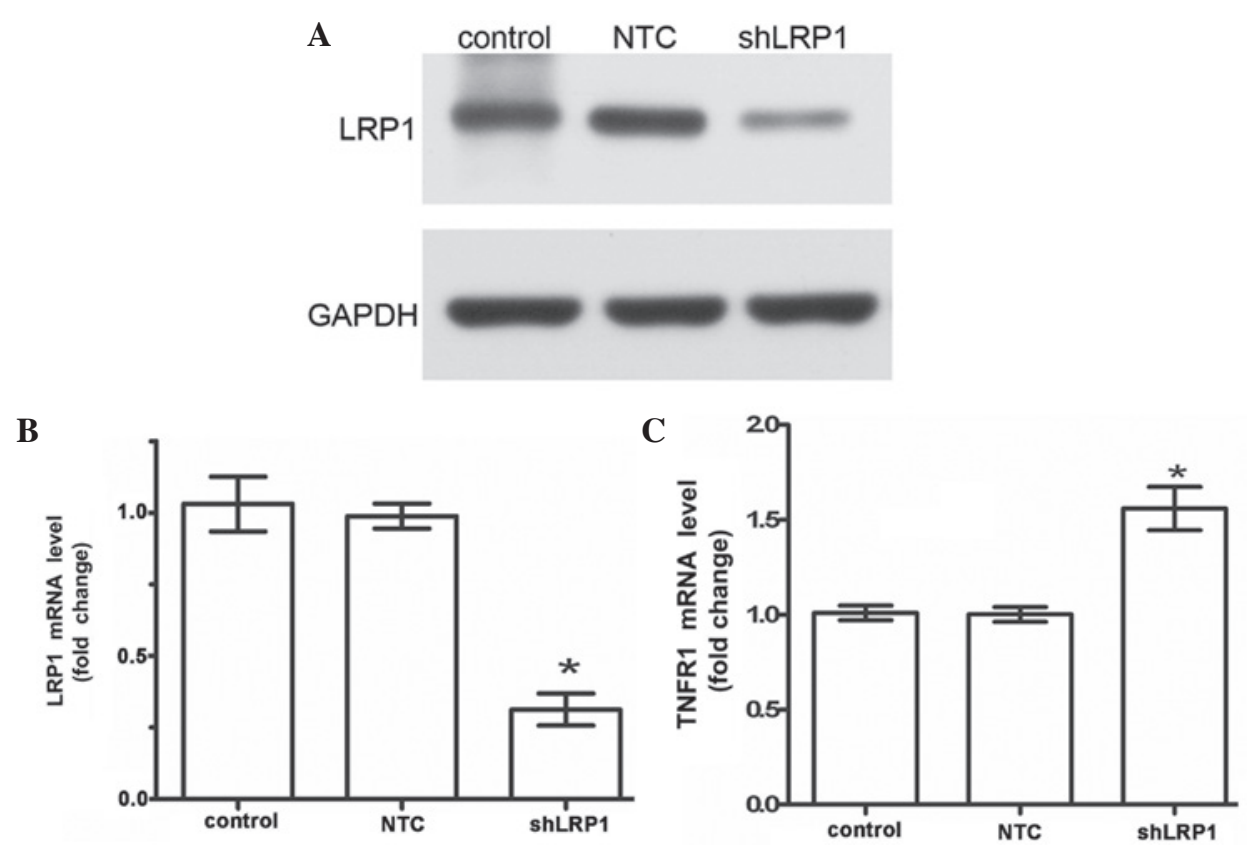

Figure 1. (A) Representative western blot used to measure the protein expression level of LRP1 at $72 \mathrm{~h}$ after transfection with a lentivirus vector expressing shRNA targeting LRP1. (B) mRNA expression levels of LRP-1 were analyzed using quantitative polymerase chain reaction (qPCR) at 48 h after shLRP1 tranfection. (C) mRNA expression levels of TNFR1 were analyzed by qPCR at $72 \mathrm{~h}$ after transfection. Values are represented as the mean \pm standard deviation. ${ }^{*} \mathrm{P}<0.05$, vs. control and NTC groups. NTC, non-targeting control; shLRP1, small hairpin RNA targeting low-density lipoprotein receptor-related protein 1; GAPDH, glyceraldehyde 3-phosphate dehydrogenase; TNFR1, tumor necrosis factor receptor 1.

tions. Briefly, the cells were fixed in $4 \%$ paraformaldehyde solution at $4^{\circ} \mathrm{C}$ for $1 \mathrm{~h}$ and washed three times with fresh phosphate-buffered saline (PBS). The slides were incubated with $50 \mu 1$ terminal deoxynucleotidyl transferase and TUNEL Reaction mixture for $1 \mathrm{~h}$ at $37^{\circ} \mathrm{C}$ in the dark. 2-(4-Amidinophenyl)-6-indolecarbamidine dihydrochloride (Beyotime Institute of Biotechnology) solution was added to stain cell nucleus for $5 \mathrm{~min}$. After washing with PBS for 5 times with 3 min interval, slides were covered with anti-fade mounting medium. Three fields were obtained from each group using a fluorescence microscope (Nikon Corporation, Tokyo, Japan).

Statistical analysis. Statistical evaluations were conducted using SPSS software (SPSS, Inc., Chicago, IL, USA). Data are presented as the mean \pm standard deviation. The statistical significance between the differences in the mean values was assessed using one-way analysis of variance, where $\mathrm{P}<0.05$ was considered to indicate a statistically significant difference. 

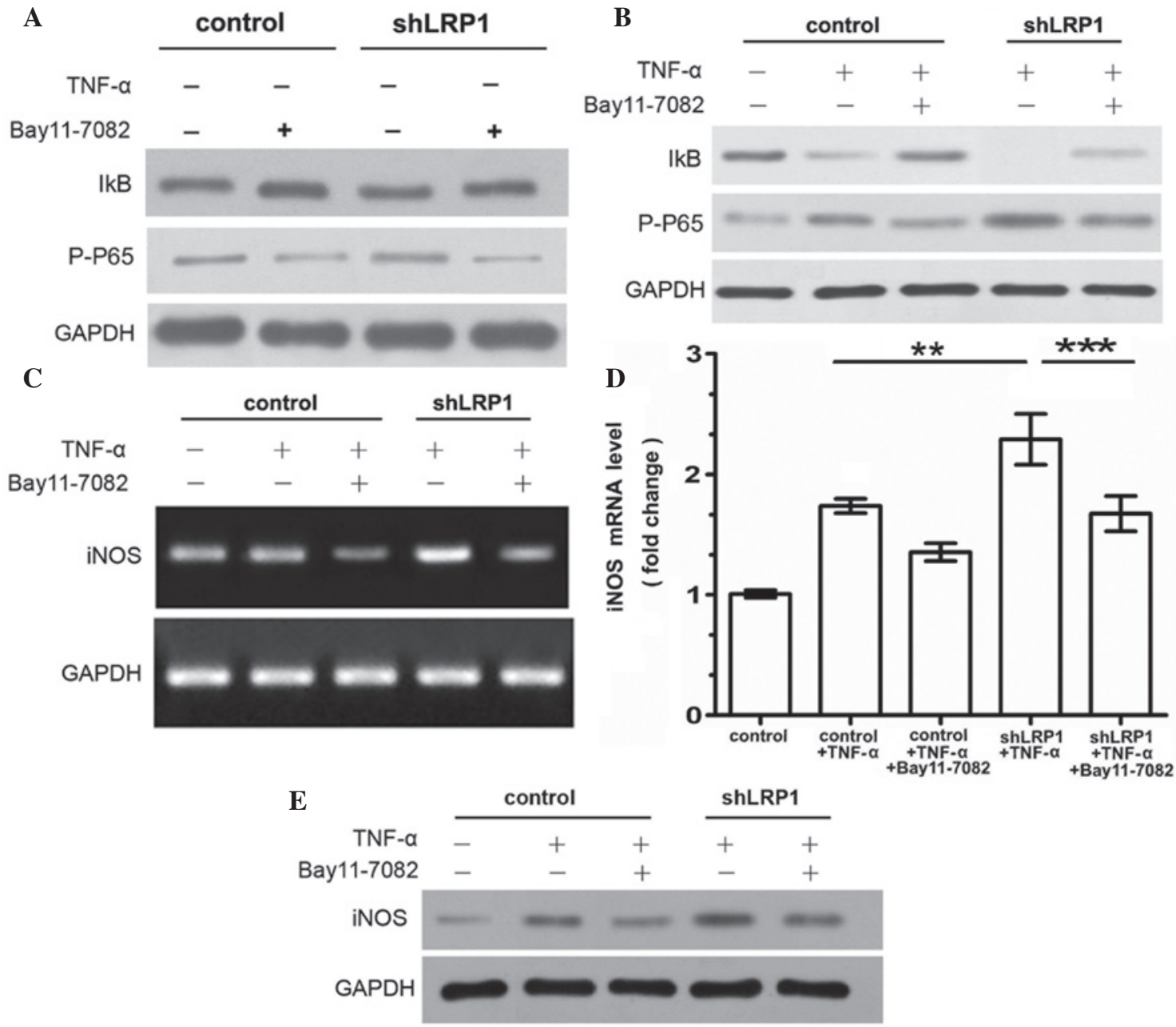

Figure 2. Effects of LRP1 on the TNF- $\alpha$-induced IкB kinase-nuclear factor- $\kappa B$ signaling pathway in chondrocytes. Equal quantities of cell extract were subjected to western blot analysis for IкB and NF- $\mathrm{B}$ B subunit p65/RelA. Control and shLRP1 chondrocytes were starved in Dulbecco's modified Eagle's medium overnight, and treated with a NF-kB inhibitor (Bay 11-7082; $10 \mu \mathrm{M}$ ) for $30 \mathrm{~min}(\mathrm{~A})$ without TNF- $\alpha$ stimulation and (B) followed by stimulation with TNF- $\alpha$ $(30 \mathrm{ng} / \mathrm{ml}$ ) for $30 \mathrm{~min}$. (C and D) mRNA and (E) protein expression levels of iNOS were assessed using quantitative polymerase chain reaction and western blot analysis, respectively. ${ }^{* *} \mathrm{P}<0.01$ and ${ }^{* * *} \mathrm{P}<0.001$. shLRP1, small hairpin RNA targeting low-density lipoprotein receptor-related protein 1 ; TNF, tumor necrosis factor; GAPDH, glyceraldehyde 3-phosphate dehydrogenase; iNOS, nitric oxide synthase.

\section{Results}

Effect of LRP1 knockdown on the chondrocytes. In order to knockdown LRP1 expression, RNA interference technology was used to transfect the chondrocytes with a lentivirus vector expressing an LRP1-targeting shRNA. The transfection efficiency was $\sim 80 \%$, as determined by fluorescence microscopy. Thus, the expression levels of LRP1 protein and mRNA were substantially knocked down to $70 \%$ of the control (control, 1.020 \pm 0.01826 ; NTC, 0.9875 \pm 0.02175 ; shLRP1, $0.3125 \pm 0.0175$; Fig. $1 \mathrm{~A}$ and B). Furthermore, the cell-surface mRNA expression levels of TNFR1 were measured using qPCR, and the TNFR1 mRNA expression levels were determined to be higher in the shLRP1 cells when compared with the control cells (control, 1.010 \pm 0.01958 ; NTC, $1.003 \pm 0.01931$; shLRP1, 1.560 \pm 0.057 ; Fig. 1C). These results indicated that the mRNA knockdown was specific for LRP1.

IKK-NF- $\kappa B$ pathway is activated by LRPI knockdown in chondrocytes. NF- $\mathrm{kB}$ is a key mediator of TNF- $\alpha$ signal transduction in chondrocytes (4). Therefore, the effect of LRP1 on
TNF- $\alpha$-induced NF- $\kappa \mathrm{B}$ activation and IкB phosphorylation was evaluated in chondrocytes using western blot analysis. ІкB binds NF- $\kappa B$, retaining it in the cytoplasm. Phosphorylation of $\mathrm{I} \kappa \mathrm{B}$ promotes $\mathrm{I} \kappa \mathrm{B}$ the degradation and release of activated $\mathrm{NF}-\kappa \mathrm{B}$. In order to evaluate the role of the IKK-NF- $\mathrm{BB}$ signaling pathway in mediating inflammation, the basal condition of the IKK-NF- $\mathrm{KB}$ pathway was determined by treating the chondrocytes with an NF-kB-specific inhibitor (Bay 11-7082) for $30 \mathrm{~min}$, with no exogenous stimulants. No statistically significant differences were observed between the control and shLRP1 groups with regard to the basal levels of the phosphory-

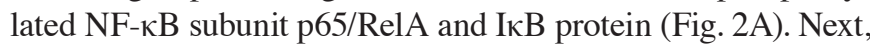
the activity of the IKK-NF-кB pathway was analyzed in chondrocytes treated with Bay 11-7082 for 30 min prior to TNF- $\alpha$ stimulation. Protein expression of phosphorylated p65/RelA was observed in the control and LRP1-knockdown cells exposed to TNF- $\alpha$, although increased levels of the phosphorylated NF- $\kappa B$ subunit p65/RelA were observed and I $\mathrm{KB}$ was undetectable in the shLRP1 cells. Furthermore, Bay 11-7082 was demonstrated to inhibit the upregulation of phosphorylated p65/RelA in the shLRP1 chondrocytes treated with TNF- $\alpha$ (Fig. 2B). 

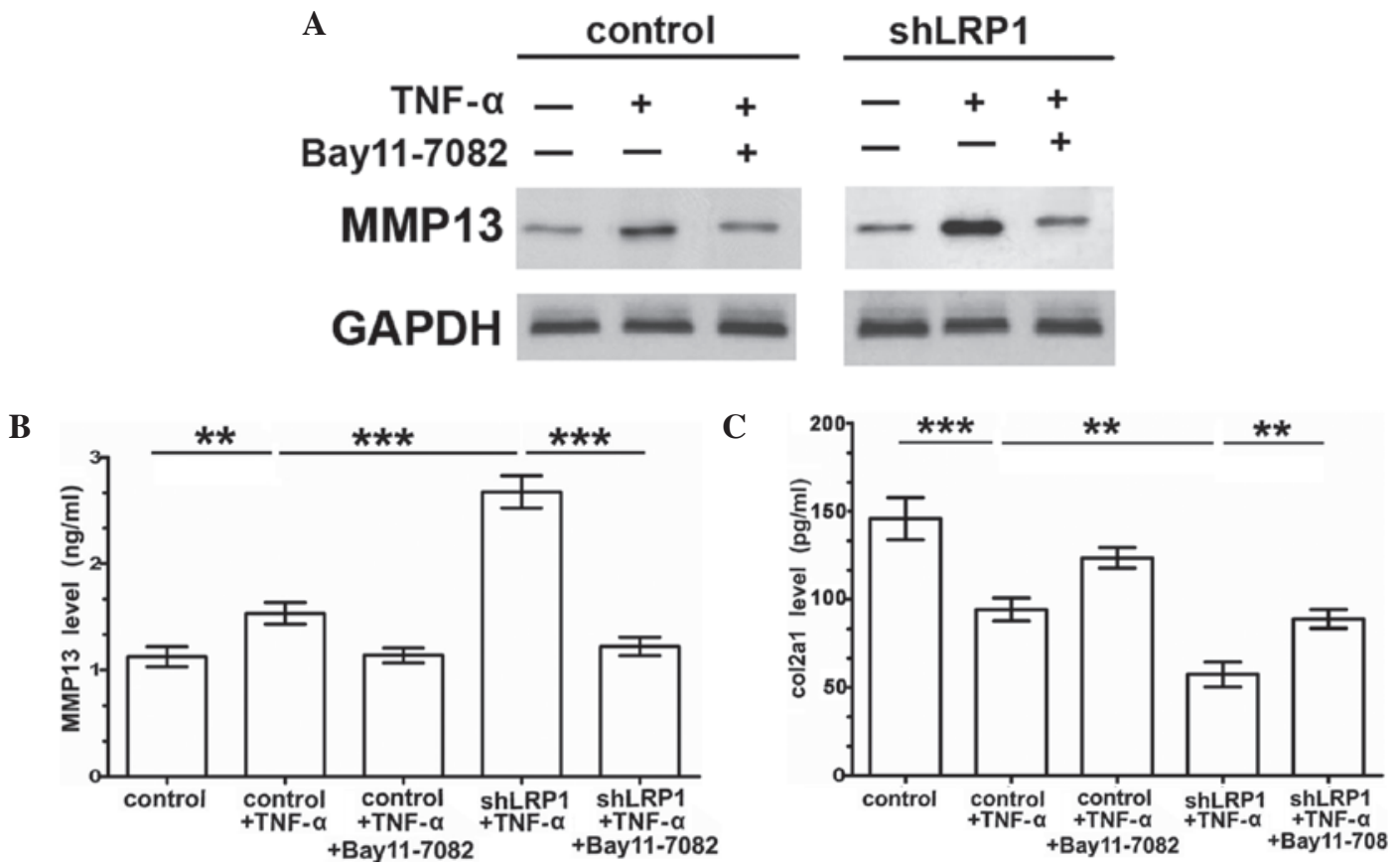

Figure 3. Effects of LRP1 on TNF- $\alpha$-induced protein expression of MMP-13 in the various chondrocyte types. Chondrocytes were pretreated with or without Bay 11-7082 $(10 \mu \mathrm{M})$ for $2 \mathrm{~h}$, followed by stimulation with or without TNF- $\alpha(30 \mathrm{ng} / \mathrm{ml})$ for $24 \mathrm{~h}$. (A) Western blot analysis was used to determine the protein expression levels. ELISA analysis showing the (B) MMP-13 and (C) COL2A1 concentration in the culture media. ${ }^{* *} \mathrm{P}<0.01$ and ${ }^{* * * *} \mathrm{P}<0.001$. shLRP1, small hairpin RNA targeting low-density lipoprotein receptor-related protein 1; TNF, tumor necrosis factor; MMP, matrix metalloproteinase; GAPDH, glyceraldehyde 3-phosphate dehydrogenase.

Knockdown of LRPI increases TNF- $\alpha$-induced expression of iNOS. Experiments were conducted to determine whether the knockdown of LRP1 promoted the enhanced transcription of iNOS, as a result of NF- $\kappa \mathrm{B}$ activation. The results revealed that the mRNA expression levels of iNOS were elevated in the shLRP1 chondrocytes treated with TNF- $\alpha$, as compared with the control cells. Furthermore, the mRNA and protein expression levels of iNOS were significantly reduced in the Bay 11-7082-pretreated shLRP1 chondrocytes exposed to TNF- $\alpha$ (shLRP1 + TNF- $\alpha, 2.29 \pm 0.12$; control + TNF- $\alpha$,

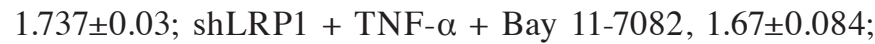
Fig. 2C-E), providing evidence for the activation of $N F-\kappa B$ in the LRP1 knockdown cells.

Knockdown of LRP1 increases TNF- $\alpha$-induced MMP-13 expression by activating the $N F-\kappa B$ pathway. The IKK-NF- $\kappa \mathrm{B}$ pathway is one of a number of key TNF- $\alpha$ signal transducers in chondrocytes, and the signaling pathway reportedly regulates the transcription of MMP-13 (4). Therefore, the effects of LRP1 on TNF- $\alpha$-induced MMP-13 expression were investigated. In the absence of exogenous stimulants, no statistically significant alterations were observed in the base protein expression levels of MMP-13 between the control and shLRP1 groups. However, MMP-13 expression was substantially increased in the shLRP1 chondrocytes stimulated with TNF- $\alpha$ (Fig. 3A). ELISA analysis was used to determine the MMP-13 expression levels in the chondrocyte culture medium. As shown in Fig. 3B, the extracellular levels of MMP-13 were increased in the TNF- $\alpha$-induced shLRP1 chondrocyte medium. Furthermore, the results indicated that the levels of MMP-13 were significantly reduced in the Bay 11-7082-pretreated shLRP1 cells that were exposed to TNF- $\alpha$ (control, 1.125 \pm 0.54 ;
shLRP1 + TNF- $\alpha, 2.675 \pm 0.088$; control + TNF- $\alpha, 1.533 \pm 0.059$; shLRP1 + TNF- $\alpha$ + Bay 11-7082, 1.221 \pm 0.05$)$. In addition, extracellular levels of COL2A1 were significantly downregulated in the TNF- $\alpha$-induced shLRP1 chondrocytes (control, 145.8 \pm 6.85 ; shLRP1 + TNF- $\alpha, 57.44 \pm 4.12$; control + TNF- $\alpha$, 94.26 \pm 3.754 ; shLRP1 + TNF- $\alpha+$ Bay 11-7082, 89.01 \pm 3.1 ; Fig. 3C), which was reversed with Bay 11-7082 pretreatment. These results indicated that LRP1 inhibited TNF- $\alpha$-induced MMP-13 expression and indirectly upregulated the extracellular levels of COL2A1 via the inhibition of NF- $\mathrm{NB}$.

Knockdown LRPI chondrocytes are sensitive to TNF- $\alpha$-induced apoptosis due to the upregulation of Bax and caspase-3 and the inhibition of $p$-Akt and Bcl-2. Since LRP1 was demonstrated to markedly affect TNF- $\alpha$-induced inflammation, the effects of LRP1 on apoptosis were investigated by analyzing the levels of Akt, Bcl-2, Bax and caspase-3 using western blot analysis and a TUNEL assay. Activation of Akt and Bcl-2 has been previously associated with the inhibition of the apoptotic cleavage of caspase-3 (14). To clarify this mechanism, chondrocytes were cultured in serum free medium for $12 \mathrm{~h}$ and treated with TNF- $\alpha$ or DMEM for an additional $2 \mathrm{~h}$. The results indicated that TNF- $\alpha$-induced shLRP1 chondrocytes exhibited significantly upregulated expression levels of caspase-3 (control, 0.1197 \pm 0.002 ; shLRP1 + TNF- $\alpha, 0.294 \pm 0.006$; control + TNF- $\alpha$, $0.117 \pm 0.003$; shLRP1, 0.224 \pm 0.003$)$ and reduced ratios of p-Akt/Akt (control, 1.5 \pm 0.049 ; shLRP1 + TNF- $\alpha, 0.71 \pm 0.051$; control + TNF- $\alpha, 1.326 \pm 0.061$; shLRP1, $0.963 \pm 0.05$; Fig. 4A) and Bcl-2/Bax (control, 3.145 \pm 0.05 ; shLRP1 + TNF- $\alpha$, 1.309 \pm 0.048 ; control + TNF- $\alpha, 2.745 \pm 0.056$; shLRP1, 1.574 \pm 0.041 ; Fig. 4B). These results indicated that LRP1 

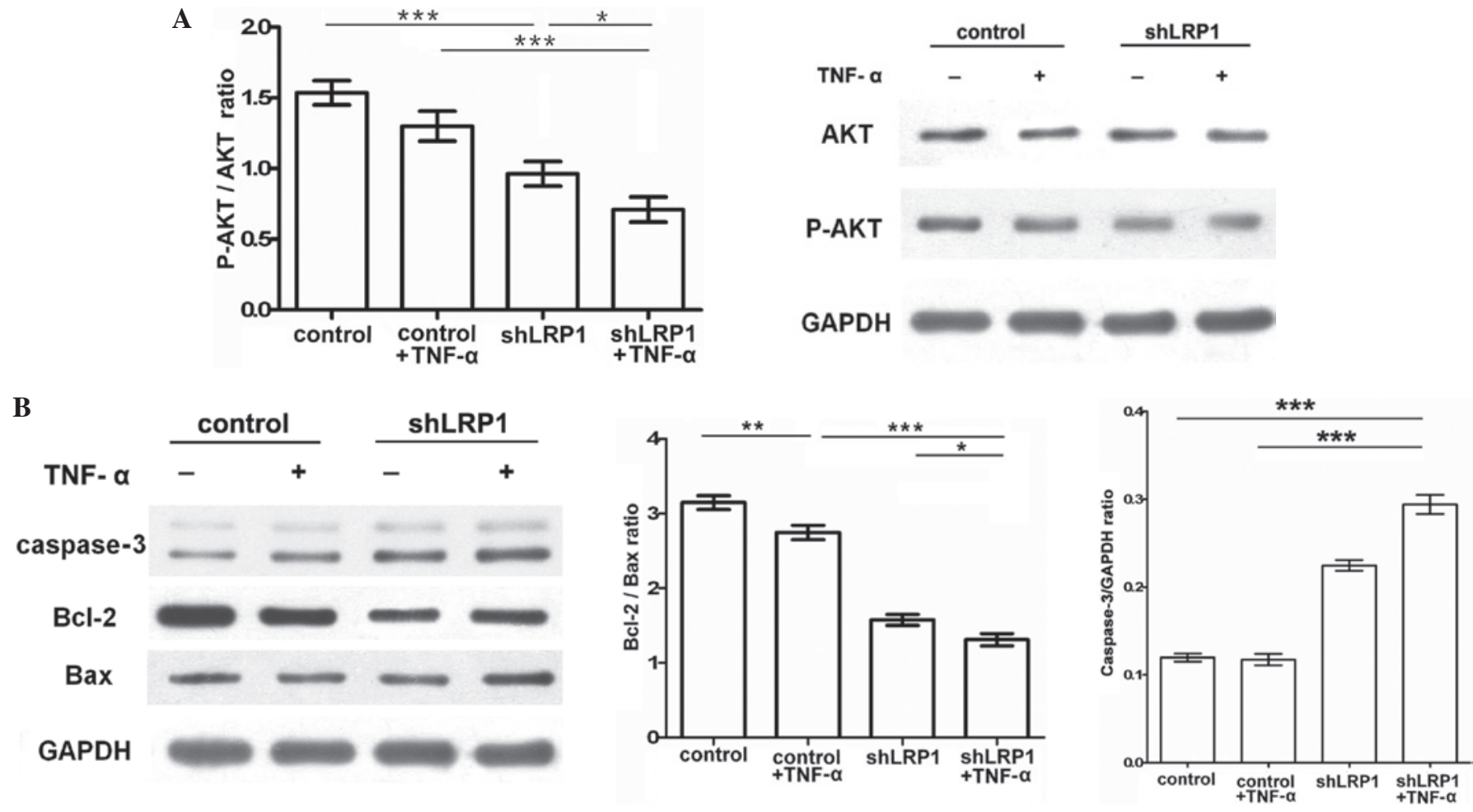

Figure 4. LRP1 knockdown inhibits TNF- $\alpha$-induced activation of the phosphoinositide 3-kinase/Akt signaling pathway in chondrocytes. Following starvation in Dulbecco's modified Eagle's medium overnight, chondrocytes were treated with or without TNF- $\alpha(30 \mathrm{ng} / \mathrm{ml})$ for $2 \mathrm{~h}$. Densitometric analyses of the bands were conducted by computerized laser densitometry and normalized against GAPDH. (A) Protein levels of total Akt, p-Akt and GAPDH were analyzed using western blot analysis. Values are expressed as the p-Akt/Akt ratio. ${ }^{*} \mathrm{P}<0.05,{ }^{* * *} \mathrm{P}<0.01$ and ${ }^{* * * *} \mathrm{P}<0.001$. (B) Protein levels of caspase-3, Bcl-2 and Bax were analyzed using western blot analysis. Values are expressed as the Bcl-2/Bax ratio and the caspase-3/GAPDH ratio. ${ }^{*} \mathrm{P}<0.05$, ${ }^{* *} \mathrm{P}<0.01$ and ${ }^{* * * *} \mathrm{P}<0.001$. p-Akt, phospho-Akt; TNF, tumor necrosis factor; shLRP1, short hairpin RNA targeting low-density lipoprotein receptor-related protein 1; GAPDH, glyceraldehyde 3-phosphate dehydrogenase; Bcl-2, B-cell lymphoma 2; Bax, Bcl-2-associated X protein.

knockdown enhanced Bax and caspase- 3 expression, while reducing Bcl-2 expression, which may serve an antiapoptotic function in chondrocytes.

To further confirm the antiapoptotic effect of LRP1 knockdown in vitro, control and shLRP1 chondrocytes were starved in DMEM overnight, after which the cells were treated with TNF- $\alpha(30 \mathrm{ng} / \mathrm{ml})$ or DMEM for $24 \mathrm{~h}$. The percentage of TUNEL-positive cells was subsequently determined microscopically. As shown in Fig. 5A and B, the proportion of apoptotic cells increased significantly in the shLRP1 chondrocytes (control, $3 \pm 0.96 \%$; shLRP1 + TNF- $\alpha, 35.11 \pm 4.53 \%$; control + TNF- $\alpha, 6.55 \pm 0.96 \%$; shLRP1, 21.25 $\pm 2.01 \%$ ).

\section{Discussion}

Low-density lipoprotein receptor-related protein 1 (LRP1) is a member of the well-studied family of endocytic receptors, which are larger than other members of the low-density lipoprotein receptor gene family, but structurally similar (7). LRP1 participates in the recognition and endocytosis of lipoproteins, in addition to recognizing a variety of non-lipoprotein ligands, including urokinase and tissue plasminogen activator, to participate in a range of physiological processes (15). As a function of endocytosis, LRP1 regulates the levels of certain matrix metalloproteinase (MMP) family members. For example, LRP regulates the catabolism and internalization of MMP-13 via a specific collagenase-3 receptor that functions as a primary binding site on cells (16).
As a key mediator of inflammatory signaling pathways (17), LRP1 is able to affect TNF- $\alpha$-induced inflammation. The results of the present study indicate that LRP1 regulates the expression of MMP-13 via the IKK/NF- $\mathrm{KB}$ pathway, which provides a mechanistic explanation for the anti-inflammatory activity of LRP1.

In order to further investigate the role of LRP1 in chondrocytes, cells were transfected with lentivirus vectors expressing LRP1-targeting shRNA, and treatment with shLRP1 was observed to markedly reduce the levels of LRP1 expression. In addition, this treatment increased the base levels of TNFR1 in the absence of exogenous stimulants (Fig. 2B), which was consistent with the results of a previous study (11). TNF- $\alpha$, a key proinflammatory cytokine whose levels are increased in the synovial fluid of patients with arthritis, has been shown to induce the expression of MMP-13 $(4,18)$.

The results of the present study indicate that the levels of phosphorylated NF-kB p65 increase in LRP1-knockdown chondrocytes in response to TNF- $\alpha$, which is also accompanied with increased expression of iNOS and MMP-13. This proposed IKK-NF- $\mathrm{kB}$ pathway mechanism is further supported by the attenuation of TNF- $\alpha$ induction in chondrocytes by the application of NF- $\mathrm{KB}$ inhibitors, such as Bay 11-7082, which

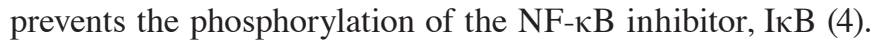
Nitric oxide (NO) is known to be a crucial inflammatory mediator in the pathogenesis of OA (19). OA cartilage generates a large quantity of NO, high levels of which have been detected in the synovial fluid and serum of patients with OA (20). 
A
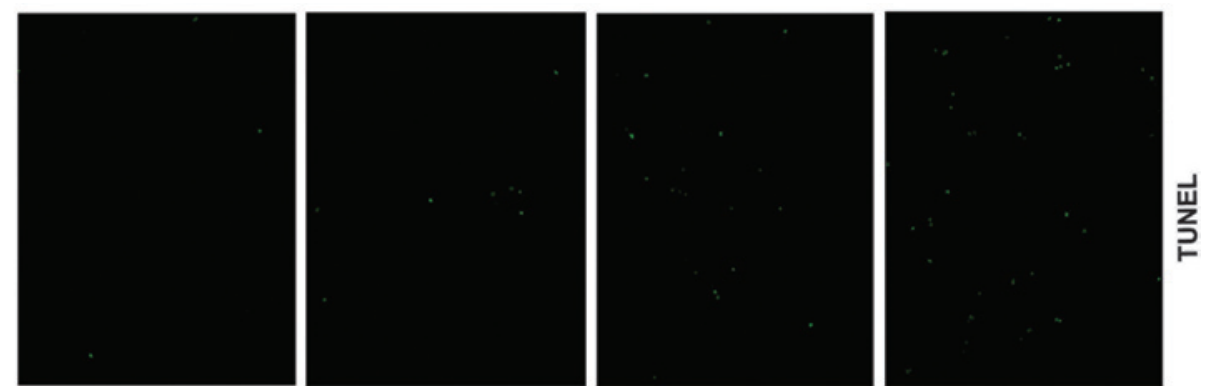

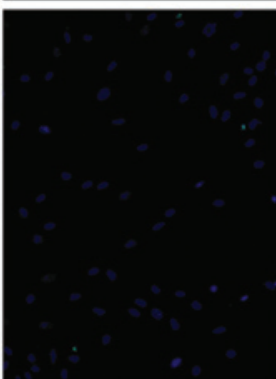

control

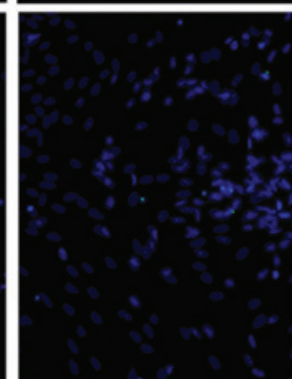

control+TNF- $\alpha$

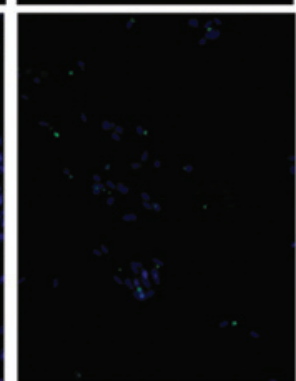

shLRP1

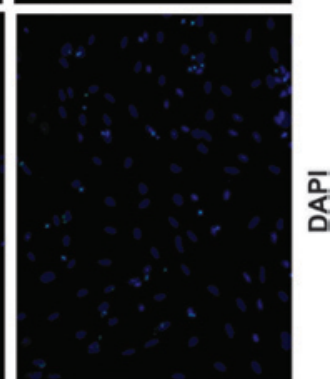

shLRP1+TNF- $\alpha$

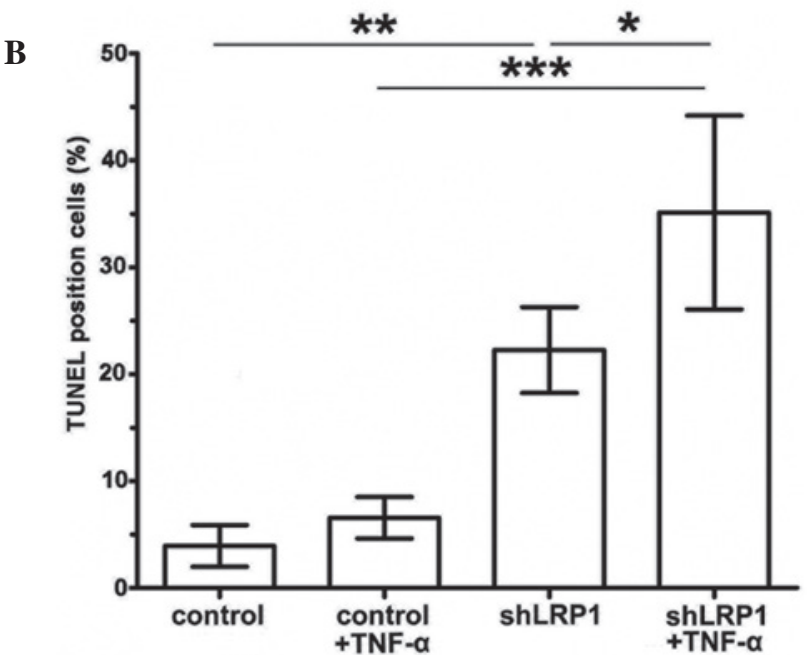

Figure 5. (A) Apoptosis of the chondrocytes was analyzed using a TUNEL assay. TNF- $\alpha$ was shown to induce apoptosis and DNA fragmentation in fluorescence micrographs of the rat chondrocytes in vitro (magnification, x200). Chondrocytes were treated with TNF- $\alpha$ (30 ng/ml) or Dulbecco's modified Eagle's medium for $24 \mathrm{~h}$. Upper panel, representative images of TUNEL staining. Lower panel, representative images showing nuclear staining of DNA (DAPI). LRP1 knockdown increased the apoptosis of chondrocytes. (B) Quantitative analysis of the percentages of TNF- $\alpha$-induced TUNEL-positive cells. Data are presented as the mean \pm standard deviation. ${ }^{*} \mathrm{P}<0.05,{ }^{* *} \mathrm{P}<0.01$ and ${ }^{* * *} \mathrm{P}<0.001$. DAPI, 4',6-diamidino-2-phenylindole; TNF, tumor necrosis factor; shLRP1, short hairpin RNA targeting low-density lipoprotein receptor-related protein 1.

Knockdown of LRP1 was observed to increase the cell-surface levels of TNFR1. By regulating the cell-surface levels of TNFR1, LRP1 can control the activity of cell signaling factors downstream of TNF- $\alpha$. Knockdown of LRP1 was demonstrated to increase the activation of the IKK-NF- $\kappa \mathrm{B}$ pathway, stimulated by TNF- $\alpha$, which was accompanied by an increase in the protein and mRNA expression levels of MMP-13. These results demonstrate the partial inhibition of MMP-13 by Bay 11-7082, indicating that LRP-1 is able to inhibit the IKK-NF- $\mathrm{B}$ pathway, which may be involved in the response of MMP-13 expression to TNF- $\alpha$. MMP-13 is a key enzyme involved in cartilage degradation, which is able to degrade type II collagen.

In chondrocytes, $\mathrm{NF}-\kappa \mathrm{B}$ signaling pathways predominate in the regulation of IL- 1 and TNF- $\alpha$-induced gene expression. Furthermore, $\mathrm{NF}-\kappa \mathrm{B}$ pathways are involved in the inhibition of
COL2A1 expression by the aforementioned cytokines (21-24). As demonstrated by the ELISA results, extracellular levels of COL2A1 were significantly downregulated in the shLRP1 chondrocytes treated with TNF- $\alpha$, which indicated that LRP1 may indirectly increase the expression of COL2A1. MMP-13-specific type II collagen cleavage products have been immunolocalized in OA cartilage $(25,26)$, and an increase in MMP-13 expression and the suppression of COL2A1 has been observed in knee joint cartilage exhibiting OA-like changes (27-29). Thus, the upregulation of MMP-13 expression may contribute to the enhanced rate of cell apoptosis observed in LRP1-knockdown chondrocytes. Furthermore, LRP1 expresses pro-survival factors in a number of cell types, including macrophages, neurons and cancer cells (30-32). Consistent with this hypothesis, LRP1 may decelerate OA progression and inhibit apoptosis in chondrocytes. 
Since various cell types are able to activate the NF- $\kappa \mathrm{B}$ pathway in response to TNF- $\alpha$ via TNFR1, the activation of NF- $\mathrm{kB}$ not only serves a crucial function in inhibiting apoptosis by inducing the upregulation of key antiapoptotic proteins, such as Bcl-2, Bcl-extra large and X-linked inhibitor of apoptosis protein $(33,34)$, but may also perform a proapoptotic function $(35,36)$. The results of the present study demonstrate that LRP1-knockdown chondrocytes exhibit increased susceptibility to cell death in response to TNF- $\alpha$, as indicated by the TUNEL assay and the nucleic morphological alterations of apoptotic chondrocytes stained with 4',6-diamidino-2-phenylindole (Fig. 5A). Furthermore, the present results identify a potential proapoptotic pathway by which knockdown of LRP1 was able to reduce p-Akt and Bcl-2 expression, while enhancing the $\mathrm{Bax} / \mathrm{Bcl}-2$ ratio and caspase-3 activation. A possible explanation may be that TNF- $\alpha$-induced apoptosis is mediated primarily through the activation of the type I receptor, which is elevated in LRP1-knockdown chondrocytes. However, p-Akt is a central regulator of cellular processes in the cytosol, one of its primary roles is to function indirectly as an antiapoptotic protein (37). Knockdown of LRP1 expression was shown to increase the levels of caspase- 3 and Bax. Active caspases serve as vital mediators in the induction of apoptosis. Furthermore, caspase- 3 is a downstream regulator of a variety of proteins, including $\mathrm{Bcl}-2$ family proteins $(30,31)$. Certain members of the $\mathrm{Bcl}-2$ family, including $\mathrm{Bcl}-2$, are able to inhibit TNF-induced apoptosis (32). Bcl-2 and Bax have been identified as key factors influencing cell susceptibility to apoptosis (31). In the present study, LRP1 knockdown chondrocytes exhibited reduced expression levels of Bcl-2 (in particular $\mathrm{Bcl}-2 / \mathrm{Bax}$ ratio), which indicates that LRP1 is able to induce cellular proliferation and exert antiapoptotic effects.

In summary, the present study demonstrated a novel function of LRP1 as an important mediator of TNF- $\alpha$ signaling, suppressing inflammation and apoptosis in articular chondrocytes. The inhibitory effects of LRP1 on apoptosis may be partially derived from the capacity of LRP1 to inhibit TNF- $\alpha$-induced apoptosis. Furthermore, the present results indicate that LRP1 exerts a protective effect in cartilage chondrocytes exposed to TNF- $\alpha$. However, the precise mechanism underlying LRP1 regulation of the cross-talk between other key pathways remains unclear, and further study is required.

\section{Acknowledgements}

This study was supported by a grant from the National Natural Science Foundation of China (no. 61308110) and by a faculty research from Wuhan University College of Medicine.

\section{References}

1. Mengshol JA, Vincenti MP, Coon CI, Barchowsky A and Brinckerhoff CE: Interleukin-1 induction of collagenase3 (matrix metalloproteinase 13) gene expression in chondrocytes requires p38, c-Jun N-terminal kinase, and nuclear factor kappaB: Differential regulation of collagenase 1 and collagenase 3 . Arthritis Rheum 43: 801-811, 2000.

2. Zhong Y, Yu W, Feng J, Fan Z and Li J: Curcumin suppresses tumor necrosis factor- $\alpha$-induced matrix metalloproteinase-2 expression and activity in rat vascular smooth muscle cells via the NF-кB pathway. Exp Ther Med 7: 1653-1658, 2014.
3. Ling $\mathrm{H}$ and Recklies AD: The chitinase 3-like protein human cartilage glycoprotein 39 inhibits cellular responses to the inflammatory cytokines interleukin-1 and tumour necrosis factor-alpha. Biochem J 380: 651-659, 2004.

4. Liacini A, Sylvester J, Li WQ, Huang W, Dehnade F, Ahmad M and Zafarullah M: Induction of matrix metalloproteinase-13 gene expression by TNF- $\alpha$ is mediated by MAP kinases, AP-1 and NF- $\kappa \mathrm{B}$ transcription factors in articular chondrocytes. Exp Cell Res 288: 208-217, 2003.

5. Campana WM, Li X, Dragojlovic N, Janes J, Gaultier A and Gonias SL: The low-density lipoprotein receptor-related protein is a pro-survival receptor in Schwann cells: Possible implications in peripheral nerve injury. J Neurosci 26: 11197-11207, 2006.

6. Zurhove K, Nakajima C, Herz J, Bock HH and May P: Gamma-secretase limits the inflammatory response through the processing of LRP-1. Sci Signal 1: ra15, 2008.

7. Herz J and Strickland DK: LRP: A multifunctional scavenger and signaling receptor. J Clin Invest 108: 779-784, 2001.

8. van Kerkhof P, Lee J, McCormick L, Tetrault E, Lu W, Schoenfish M, Oorschot V, Strous GJ, Klumperman J and Bu G: Sorting nexin 17 facilitates LRP recycling in the early endosome. EMBO J 24: 2851-2861, 2005.

9. Herz J: The LDL receptor gene family: (un)expected signal transducers in the brain. Neuron 29: 571-581, 2001.

10. Overton CD, Yancey PG, Major AS, Linton MF and Fazio S: Deletion of macrophage LDL receptor-related protein increases atherogenesis in the mouse. Circ Res 100: 670-677, 2007.

11. Gaultier A, Arandjelovic S, Niessen S, Overton CD, Linton MF, Fazio S, Campana WM, Cravatt BF III and Gonias SL: Regulation of tumor necrosis factor receptor- 1 and the IKK-NF- $\mathrm{BB}$ pathway by LDL receptor-related protein explains the antiinflammatory activity of this receptor. Blood 111: 5316-5325, 2008.

12. Gaultier A, Arandjelovic S, Li X, et al: A shed form of LDL receptor-related protein-1 regulates peripheral nerve injury and neuropathic pain in rodents. J Clin Invest 118: 161-172, 2008.

13. Hu DN, Yang PY, Ku MC, Chu CH, Lim AY and Hwang MH: Isolation and cultivation of human articular chondrocytes. Kaohsiung J Med Sci 18: 113-120, 2002.

14. Hermann C, Assmus B, Urbich C, Zeiher AM and Dimmeler S: Insulin-mediated stimulation of protein kinase Akt: A potent survival signaling cascade for endothelial cells. Arterioscler Thromb Vasc Biol 20: 402-409, 2000.

15. Gaultier A, Simon G, Niessen S, Dix M, Takimoto S, Cravatt BF III and Gonias SL: LDL receptor-related protein 1 regulates the abundance of diverse cell-signaling proteins in the plasma membrane proteome. J Proteome Res 9: 6689-6695, 2010.

16. Barmina OY, Walling HW, Fiacco GJ, Freije JM, López-Otín C, Jeffrey JJ and Partridge NC: Collagenase-3 binds to a specific receptor and requires the low density lipoprotein receptor-related protein for internalization. J Biol Chem 274: 30087-30093, 1999.

17. Raggatt LJ, Jefcoat SC Jr, Choudhury I, Williams S, Tiku M and Partridge NC: Matrix metalloproteinase-13 influences ERK signalling in articular rabbit chondrocytes. Osteoarthritis Cartilage 14: 680-689, 2006.

18. Manicourt DH, Poilvache P, Van Egeren A, Devogelaer JP, Lenz ME and Thonar EJ: Synovial fluid levels of tumor necrosis factor alpha and oncostatin M correlate with levels of markers of the degradation of crosslinked collagen and cartilage aggrecan in rheumatoid arthritis but not in osteoarthritis. Arthritis Rheum 43: 281-288, 2000.

19. Henrotin Y, Kurz B and Aigner T: Oxygen and reactive oxygen species in cartilage degradation: Friends or foes? Osteoarthritis Cartilage 13: 643-654, 2005.

20. Henrotin Y and Kurz B: Antioxidant to treat osteoarthritis: Dream or reality? Curr Drug Targets 8: 347-357, 2007.

21. Séguin CA and Bernier SM: TNFalpha suppresses link protein and type II collagen expression in chondrocytes: Role of MEK1/2 and NF-kappaB signaling pathways. J Cell Physiol 197: 356-369, 2003.

22. Robbins JR, Thomas B, Tan L, Choy B, Arbiser JL, Berenbaum F and Goldring MB: Immortalized human adult articular chondrocytes maintain cartilage-specific phenotype and responses to interleukin-1beta. Arthritis Rheum 43: 2189-2201, 2000.

23. Murakami S, Lefebvre V and de Crombrugghe B: Potent inhibition of the master chondrogenic factor Sox 9 gene by interleukin-1 and tumor necrosis factor-alpha. J Biol Chem 275: 3687-3692, 2000.

24. Sitcheran R, Cogswell PC and Baldwin AS Jr: NF-kappaB mediates inhibition of mesenchymal cell differentiation through a posttranscriptional gene silencing mechanism. Genes Dev 17: 2368-2373, 2003. 
25. Tetlow LC, Adlam DJ and Woolley DE: Matrix metalloproteinase and proinflammatory cytokine production by chondrocytes of human osteoarthritic cartilage: Associations with degenerative changes. Arthritis Rheum 44: 585-594, 2001.

26. Wu W, Billinghurst RC, Pidoux I, Antoniou J, Zukor D, Tanzer M and Poole AR: Sites of collagenase cleavage and denaturation of type II collagen in aging and osteoarthritic articular cartilage and their relationship to the distribution of matrix metalloproteinase 1 and matrix metalloproteinase 13. Arthritis Rheum 46: 2087-2094, 2002.

27. Neuhold LA, Killar L, Zhao W, Sung ML, Warner L, Kulik J, et al: Postnatal expression in hyaline cartilage of constitutively active human collagenase-3 (MMP-13) induces osteoarthritis in mice. J Clin Invest 107: 35-44, 2001.

28. Aigner T, Vornehm SI, Zeiler G, Dudhia J, von der Mark K and Bayliss MT: Suppression of cartilage matrix gene expression in upper zone chondrocytes of osteoarthritic cartilage. Arthritis Rheum 40: 562-569, 1997.

29. Aigner T, Zien A, Gehrsitz A, Gebhard PM and McKenna L: Anabolic and catabolic gene expression pattern analysis in normal versus osteoarthritic cartilage using complementary DNA-array technology. Arthritis Rheum 44: 2777-2789, 2001.

30. Cui N, Li S, Zhao X, Zhang T, Zhang C, Yu L, Zhu Z and Xie K: Expression of Bcl-2, Bax and Caspase-3 in nerve tissues of rats chronically exposed to 2,5-hexanedione. Neurochem Res 32: 1566-1572, 2007.
31. Postolow F, Fediuk J, Nolette N, Hinton M and Dakshinamurti S: Hypoxia and nitric oxide exposure promote apoptotic signaling in contractile pulmonary arterial smooth muscle but not in pulmonary epithelium. Pediatr Pulmonol 46: 1194-1208, 2011.

32. Rath PC and Aggarwal BB: TNF-induced signaling in apoptosis. J Clin Immunol 19: 350-364, 1999.

33. Graham B and Gibson SB: The two faces of NFkappaB in cell survival responses. Cell Cycle 4: 1342-1345, 2005.

34. Karin M and Lin A: NF-kappaB at the crossroads of life and death. Nat Immunol 3: 221-227, 2002.

35. Luo JJ, Li CY, Liu S, Yu W, Tang SY, Cai HL and Zhang Y: Overexpression of Helicobacter pylori VacA N-terminal fragment induces proinflammatory cytokine expression and apoptosis in human monocytic cell line through activation of NF-kB. Can J Microbiol 59: 523-533, 2013.

36. Liu X, Xiao Q, Zhao K and Gao Y: Ghrelin inhibits high glucose-induced PC12 cell apoptosis by regulating TLR4/NF-KB pathway. Inflammation 36: 1286-1294, 2013.

37. Swaminathan JK, Khan M, Mohan IK, Selvendiran K, Niranjali Devaraj S, Rivera BK and Kuppusamy $P$ : Cardioprotective properties of Crataegus oxycantha extract against ischemia-reperfusion injury. Phy tomedicine 17: 744-752, 2010 . 